\title{
Use of superior mesenteric vein for renal transplant venous outflow in a patient with extensive inferior vena cava thrombosis
}

\author{
Nalaka Gunawansa $^{1,2}$, Ajay Sharma ${ }^{2,3}$, Ahmed Halawa ${ }^{2,4}$ \\ ${ }^{1}$ National Institute of Nephrology Dialysis and Transplant, Sri Lanka \\ ${ }^{2}$ Faculty of Health and Science, Institute of Learning and Teaching, University of Liverpool, UK \\ ${ }^{3}$ Royal Liverpool University Hospital, Liverpool, UK \\ ${ }^{4}$ Sheffield Teaching Hospitals, Sheffield, UK.
}

Key words: Renal transplantation; vena cava; mesenteric vein; thrombosis

\section{Introduction}

The routine vascular anastomosis in renal transplantation is performed to the recipient iliac vessels. In paediatric recipients (weight $<15 \mathrm{~kg}$ ) and certain re-transplants, venous and arterial anastomosis may be performed to the inferior vena cava (IVC) and aorta respectively $(1,2)$. Therefore, when the common iliac veins (CIV) and IVC are both affected by systemic thrombosis, possible venous outflow channels for transplantation are limited and challenging. It may often result in significant delays or even being denied access to transplantation due to technical feasibility as well as potential thrombotic complications post-transplant.

\section{Case report}

S.N was a 28-year-old female in end stage renal failure (ESRF), with systemic lupus erythematosus and recurrent lower limb deep vein thrombosis since adolescence. She was on long-term anticoagulation (warfarin) since the age of 16 and on maintenance haemodialysis (right sided subclavian catheter) for 14 months. Her mother (47 years), came forward for live donation.

\section{Assessment}

Pre-operative duplex imaging of the patient showed an occluded left CIV. The right CIV though patent, showed monophasic flow with evidence of prior thrombotic disease and recanalization. Further imaging with magnetic resonance angiography confirmed extensive thrombosis of left CIV and infra-hepatic IVC (Figure 1, 2). There was extensive collateral formation along the left inferior epigastric, lumbar and retroperitoneal veins. The superior mesenteric vein (SMV) and portal vein were intact. Although the right CIV was patent, it appeared to be draining in to retroperitoneal collaterals. The upstream thrombus and complete occlusion of the IVC and monophasic flow within the right CIV were considered deterrents to successful graft implantation. The

Correspondence: Nalaka Gunawansa

E-mail: vascular@drnalakagunawansa.com

Received: 09-05-2018 Accepted: 19-06-2018

(iDhttps://orcid.org/0000-0003-0098-3855

DOI: http://doi.org/10.4038/sljs.v36i2.8516

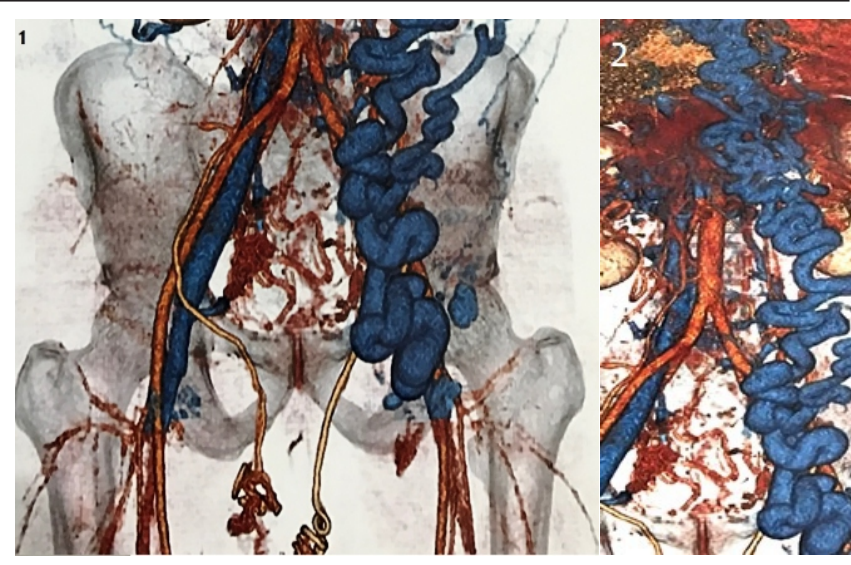

Figure 1. Magnetic Resonance Angiogram of the recipient Figure 2. Extensive collateral formation around occluded iliac veins and vena cava

patent portal-mesenteric system was considered as a potential alternative. Detailed counselling was done for both the donor and recipient regarding the possible outcomes and long-term benefits of transplantation versus haemodialysis.

The donor renal angiogram showed two divergent arteries to left and a single artery to right kidney. Differential renal functions were; Left $53 \%$, right $47 \%$. The right kidney was selected for donation.

\section{The recipient operation}

Anticoagulation was changed from warfarin to enoxaparin, 4 days before surgery. The last dose of enoxaparin was given on the eve of surgery, while keeping the patient on graduated compression stockings throughout her hospital stay. A Midline laparotomy was done, and preliminary vascular assessment was performed. The IVC and aorta were exposed by medial rotation of the right colon; Cattell-Braasch manoeuvre. The IVC and left CIV were occluded with palpable hard thrombi and severe inflammation in the perivenous tissue. The IVC thrombus extended beyond the confluence with native renal veins. The portal and superior mesenteric veins were patent and unaffected by thrombotic process. This was confirmed by intra-operative on-table duplex imaging.

A right laparoscopic donor nephrectomy was performed in conventional fashion. The retrieved kidney was cold perfused 


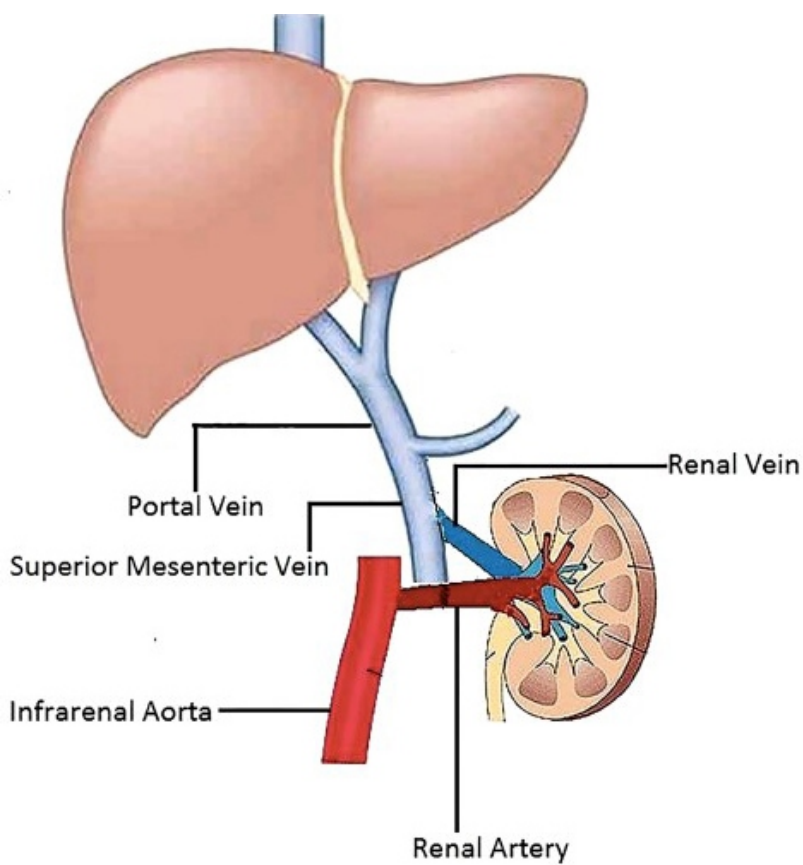

Figure 3. Schematic diagram of the vascular reconstruction

using histidine-tryptophan-ketogluterate solution in the backtable. The donor renal artery was short and was reconstructed with an extension venous graft (recipient right reversed great saphenous vein). The allograft vein was anastomosed to the proximal SMV (Figure 3); end-to-side configuration using $5 / 0$ polypropylene. The reconstructed renal artery was anastomosed end-to-side to the aorta also with $5 / 0$ polypropylene. The total warm ischaemia time was 21 minutes and cold ischaemia time was 34 minutes. Immediate reperfusion was done and showed excellent graft perfusion with minimal blood loss or systemic effects on the recipient. The ureter was anastomosed to the native right ureter (end-toside), over a 5 French ureteric stent using 6/0 polydiaxone suture.

\section{Peri-operative period}

The immunosuppression was in keeping with that for a 'lowimmunological risk' transplant, consisting of basiliximab induction, tacrolimus, mycophenolate mofetil and prednisolone. Prophylactic intravenous antibiotics were continued for 72 hours after surgery considering the extent of surgical dissection. The patient was extubated immediately after surgery and was managed in the intensive care isolation unit as for a standard transplant recipient. Oral feeding was restricted to liquids in the first 24 hours and solids were introduced from day-02. Subcutaneous enoxaparin $20 \mathrm{mg}$ daily was continued from day- 01 .

The allograft showed immediate function with satisfactory diuresis, achieving normal serum creatinine levels by day-03 Warfarin was started at this time (day-03) and was continued along with enoxaparin until therapeutic levels were achieved (day-07). At this time, enoxaparin was discontinued, and warfarin was continued at the same dose. Duplex imaging (day-01, day-04), showed excellent graft perfusion and venous drainage. She was discharged on day-08 with normal graft function (serum creatinine $1.1 \mathrm{mg} / \mathrm{dl}$ ).

\section{Post-operative care}

Initial post-operative visits showed sustained graft function (serum creatinine $0.9-1.1 \mathrm{mg} / \mathrm{dl}$ ). On day-27, she was admitted with elevated serum creatinine, $1.9 \mathrm{mg} / \mathrm{dl}$. Duplex scan showed high arterial resistive indices (0.87-0.89) with normal venous drainage. The blood tacrolimus level was 9.3 $\mathrm{ng} / \mathrm{ml}$. A biopsy was not performed due to on-going anticoagulation and was treated empirically with MethylPrednisolone Pulsing. Graft function returned to normal with treatment and has been sustained since. Presently (15 months post-operative), she maintains satisfactory graft function (Serum creatinine $1.2 \mathrm{mg} / \mathrm{dl}$ ) and remains in good health.

\section{Discussion}

In the absence of patent iliac veins for allograft venous drainage, the alternatives are infra or supra hepatic IVC, native renal veins after native nephrectomy or mesenteroportal veins $(3,4)$. Successful implantation to the portal vein, SMV and splenic vein in paediatric recipients with deceased donor grafts has been reported with reasonable success in small numbers (5).

We did not use the right CIV due to the extensive upstream thrombosis in the IVC and monophasic flow on duplex. Extension of the IVC thrombus beyond the confluence with renal veins precluded implantation in to the native renal veins. The short length of the right donor renal vein did not allow us to reach the portal vein, while using the aorta for arterial anastomosis. The inferior mesenteric vein although patent, appeared too delicate, thin walled and small. Hence it was decided to use the larger SMV as the venous outflow. Postoperative clinical and duplex surveillance did not show any impact on graft function, native liver function or portal venous flow.

\section{Conclusion}

Renal transplantation offers the best outcomes for patients in ESRF. Thrombosis of IVC and CIV should not be considered contra-indications to renal transplantation thereby denying such patients the chance for a transplant. While maintaining therapeutic anticoagulation to prevent recurrent thrombosis, alternate venous drainage routes such as the portal-mesenteric system should be explored where feasible. Although deceased donor transplants allow extra length of graft vessels to perform complex vascular reconstruction, live donor transplants are limited by the short length of graft artery and vein. Nevertheless, with meticulous planning and care, live donor transplants can also be performed for the selected individual patients. Available short-term results have shown encouraging outcomes with excellent graft function. 
Explicit informed written consent has been obtained from the patient regarding the academic publication of this article with relevant details.

All authors disclose no conflict of interest. The study was conducted in accordance with the ethical standards of the relevant institutional or national ethics committee and the Helsinki Declaration of 1975, as revised in 2000

\section{References}

1. Watson CJE, Friend PJ. Chapter - 11 Surgical Techniques of Kidney Transplantation. In: Morris P, Knechtle SJ, editors. Kidney Transplantation-Principles and Practice. 2014. page $161-75$.

2. Adams J, Gudemann C, Tonshoff B, Mehls O, Wiesel M. Renal transplantation in small children--a comparison between surgical procedures. Eur. Urol. 2001;40:552-6.

https://doi.org/10.1159/000049835
3. Pirenne J, Benedetti E, Kashtan CE, Llédo-Garcia E, Hakim N, Schroeder CH, et al. Kidney transplantation in the absence of the infrarenal vena cava. Transplantation 1995;59:1739-42.

https://doi.org/10.1097/00007890-199506270-00018

4. Aguirrezabalaga J, Novas S, Veiga F, Chantada V, Rey I, Gonzalez $\mathrm{M}$, et al. Renal transplantation with venous drainage through the superior mesenteric vein in cases of thrombosis of the inferior vena cava. Transplantation 2002;74:413-5.

https://doi.org/10.1097/00007890-200208150-00022

5. Millan M, Caicedo LA, Villegas JI, Serrano O, Caicedo L, Duque $\mathrm{M}$, et al. Case report of cadaveric kidney transplantation with renal-portal venous drainage: A feasible way for a venous drainage in a complex generalized thrombosed vessels setting. Int. J. Surg. Case Rep. 2016;28:192-5.

https://doi.org/10.1016/j.ijscr.2016.09.047

\section{Learning Points:}

- IVC thrombosis and thrombophilias are not a contra-indication for renal transplantation

- The portal-mesenteric venous system is often spared in systemic thrombotic disease

- Successful transplantation can be done using the portal-mesenteric system with full anti-coagulation. 